\title{
The Social and Cultural Implications of the Egg Freezing Policy in China
}

\author{
Chenyun Shao ${ }^{1}$ \\ ${ }^{1}$ The Vivian Webb School, Claremont, California, United States \\ Correspondence: Chenyun Shao, The Vivian Webb School, Claremont, California, United States.
}

Received: September 14, 2020

Accepted: October 13, $2020 \quad$ Available online: October 22, 2020

doi:10.11114/ijsss.v8i6.5014

URL: https://doi.org/10.11114/ijsss.v8i6.5014

\begin{abstract}
The technology of egg freezing has become increasingly popular and has caused heated scholarly debates. However, most scholarships focus on egg freezing exclusively in the United States. This paper fills in the gap by investigating the social and cultural implications of the egg freezing policy in China. This paper first examines feminist rhetoric used by both sides in the global egg freezing debate. The paper then introduces the current Chinese egg freezing policy. This section addresses the question, "Why does the Chinese government implement the current egg freezing policy?" by analyzing how the Chinese culture views reproduction and single women. By analyzing egg freezing in context of the Chinese culture, I concluded that the older and younger generations have different views on egg freezing. Finally, I share the results from a survey that I designed and distributed to see how Chinese people perceive egg freezing. I found that most respondents support universal access to egg freezing in China but have questions on the risks and disadvantages of egg freezing. Many respondents also perceive egg freezing as a technology to "preserve/guarantee motherhood" for women, which ironically serves to pressure women into motherhood instead of giving them true reproductive freedom.
\end{abstract}

Keywords: egg freezing policy in China, the debate of egg freezing, the Chinese culture, Chinese single women, reproduction

\section{Introduction}

\subsection{The Concept of Egg Freezing in China}

The concept of egg freezing remained unfamiliar to most Chinese until Xu Jinglei, a famous Chinese actress, shared her egg freezing experience in her interview with the Chinese lifestyle magazine Vista in 2015. Xu Jinglei contended that she went to the United States to freeze nine of her eggs in 2013 when she was thirty-nine years old. She told the interviewer that she wished she had done the procedure sooner and described it as "the world's only remedy for regret." She also shared her experience on Weibo, a Chinese microblogging platform, allowing more Chinese people to learn about the technology of egg freezing (Hilgers, 2015).

A few years after Xu Jinglei's interview, Teresa Xu (unrelated to Xu Jinglei), a thirty-one-year-old single Chinese woman, went to Beijing Obstetrics and Gynecology Hospital to consult on freezing her eggs. Instead of receiving professional advice, Ms.Xu's doctor told her to get married soon and have a kid naturally. Ms. Xu was unsatisfied with the result of the consultation and being denied access to egg freezing, she sued the hospital for violating her human rights (Anonymous, 2019). Ms. Xu stated after her first court hearing, "Whether I choose to have a child or not, I should have the right to choose. Many women don't know that they can freeze their eggs. When their own childbearing pressure and workplace pressure overlap, they may not know there is a way to solve the problem" (Kuo, 2019). She also said that unlike the famous Chinese actress, going abroad to freeze her eggs was too expensive for her. But the spokesperson of Beijing Obstetrics and Gynecology Hospital stated that the hospital was merely complying with the government's regulations on assisted reproductive technologies (Anonymous, 2019). Being the first court case in China on egg freezing, the case gained lots of attention. The day after her first hearing, a hashtag related to Ms. Xu's case had around nine million views and thousands of comments on Weibo. Many Chinese supported Ms. Xu on Weibo and questioned why single women could not freeze their eggs in China. Legal experts said they did not have high hopes that Ms. Xu would win her lawsuit, but that the publicity and the acceptance of her case by the Chinese court is already a win (Kuo, 2019)

\subsection{Definition of the Technology of Egg Freezing}

Egg freezing, also known as oocyte cryopreservation, is a process in which a woman's eggs are extracted, frozen and stored to preserve the woman's reproductive potential. In the first step of the egg freezing process, a fertility specialist 
performs an assessment of the ovarian reserve to estimate the potential yield of oocytes prior to ovarian stimulation cycle. The assessment includes blood tests and pelvic ultrasound. The results help determine the necessary dose of medications to stimulate egg production. Then, ovarian stimulation is carried out using injectable hormonal medications. After the stimulation, the oocytes and the surrounding fluid in the ovarian follicles are extracted vaginally while the patient is under sedation. The maturity of the extracted eggs is assessed under the microscope, and the mature eggs are cryopreserved. Vitrification is the best method currently used for cryopreserving eggs, and this is achieved by the ultra-rapid cooling of the eggs into liquid nitrogen where they are stored. In 1986, the first human baby was birthed using a frozen oocyte. With the advancement of technology in the past few years, the overall success rate of eggs surviving the freezing process has greatly improved. In 2013, egg freezing is no longer labeled an experimental procedure by the American Society for Reproductive Medicine (UCLA).

\subsection{Area of Study and Objectives of the Research}

This paper focuses on the area of reproductive rights and how the technology of egg freezing enables and restricts women's reproductive rights. Through reading scholarly works on professional research websites, I found that most scholarship on egg freezing is focused on western countries such as the United States and the United Kingdom. There are no scholarly articles on Jstor and only one scholarly article on Google Scholar that centers on egg freezing in China. This void of scholarly discussions has left many questions unanswered such as how egg freezing is viewed in China, and why does the Chinese government implement policies that ban egg freezing from most Chinese women. In this paper, my objective is to answer this question: What are the social and cultural implications of China's policy on egg freezing? By answering this question and analysing the egg freezing debate in context of the Chinese culture, I hope to inform more Chinese people on women's reproductive rights and encourage scholars to do more feminist research centered on China and other Asian countries.

\subsection{Research Methods}

This paper is a qualitative study of the egg freezing policy in China. In the first part of the paper, I situate my analysis of egg freezing in China within the debates over egg freezing, focusing in particular on how feminism is deployed by those who both support and oppose the procedure. In the second part of the paper, I discuss egg freezing in the context of Chinese culture, gender, and reproduction. I include in depth discussion of statistics and evidence on the trend of marriage in China and how single women are treated. At the end of the second section, I bring together egg freezing and the Chinese cultural ideas and practices. The analysis addresses how these cultural ideas and practices inform egg freezing as a practice in China. In the third part of the paper, I discuss a survey on egg freezing that I designed and distributed to Chinese people in my network. The methodology section addresses the process of designing the questions, selecting the population, distributing the survey and data analysis. Finally, I analyze the collected data and show trends among survey respondents' positions on egg freezing. In this paper, I argue that egg freezing is a complex technology that seems to open up reproductive freedom but can actually be used to restrict women's reproductive rights. By analyzing the egg freezing debate within the context of Chinese culture, I encourage Chinese women to have a deeper understanding of egg freezing and make their own reproductive decisions instead of simply following the current Chinese egg freezing policy.

\section{Literature Review: The Place of Feminism in Debates on Egg Freezing}

Egg freezing is a hotly debated topic among scholars, and feminist approaches are utilized by those who support and oppose egg freezing. This section analyzes the debate of egg freezing on a global scale and specifically the feminist rhetoric used by both sides.

\subsection{Arguments Supporting Egg Freezing}

The American Society for Reproductive Medicine (ASRM) took off the experimental label on egg freezing in early 2013 so women who need and are willing to pay for egg freezing can use the technology freely (Pinto, 2017). With the label lifted, some scholars argue that egg freezing is beneficial for helping women solve the dilemma of choosing between a successful career and motherhood (Schulte, 2014). Scholars have provided two feminist arguments in favor of egg freezing: egg freezing makes reproduction equal for men and women, and that egg freezing allows women to focus on their careers before becoming a mother. Leslie Mitch argues that the nature of reproduction puts men and women on unequal grounds. Mitch explains that a man has almost a lifetime to become a father, but a woman's reproductive prime only lasts for around a decade, which coincides with the time for her to get an education and start a career (Mitch, 2007). One study carried out by Seema Mohapatra, a scholar in the field of public health law, found that women lose eighty eight percent of their maximum ovarian reserve by the age of thirty and ninety seven percent by the age of forty. And a woman's fertility drops from eighty six percent at age twenty to fifty two percent at age thirty-five, thirty six percent at age forty and five percent at age forty-five (Mohapatra, 2013). According to Shekdi-Rafid, egg freezing is an option that addresses women's fast declining fertility and puts them on equal ground with men in the reproductive arena. With more women entering the workforce, the age at which women first give birth continues to rise in Western countries. However, female 
fertility begins to decline in their thirties. In this case, Shekdi-Rafid states that women can use egg freezing to pause the biological clock. The technology allows women to exercise more control over their lives by postponing motherhood when they chose to. Women will be able to make conscious, voluntary, and independent decisions when they are provided with the relevant medical data. (Shekdi-Rafid et al., 2012). Mohapatra argues that some women see this new ability to control when they will have children as the ultimate step to gender equality, because egg freezing allows them to build their careers without worrying about having kids in their short prime reproductive years, something men are free from. Even women who do not know if they will have kids at all use egg freezing, because it gives them more time to consider the options of whether to have kids (Mohapatra, 2013).

Furthermore, Feminists point out that many women want to focus on their careers before becoming mothers (Mohapatra, 2013). However, the reality is harsh for many women. The intense work culture and competitive environment in companies makes pregnancy a difficult option for ambitious young women. High tech firms with high paying jobs are becoming notoriously filled with white and Asian males according to their own internal audits. Women hold few high positions. The work cultures encourage long hours every day and many employees sleep under their desks. Yahoo employees once wore t-shirts saying, "ninety hours a week and loving it!" (Schulte, 2014). This kind of work culture, according to the Anita Borg Institute for Women in Technology, "is sending the message that those who have family responsibilities need not apply" (Schulte, 2014). Egg freezing helps women who want to be mothers preserve their fertility and allow them to freely compete with their male counterparts. Some big companies have already been providing egg freezing to female employees. Pinto states that both Facebook and Apple stated that they were including egg freezing in their employee's total benefit packages in 2014, giving female employees the options to control their fertility (Pinto, 2017).

Aside from these two feminist approaches, Mohapatra wrote that egg freezing also enables women to have more time to find a suitable partner whom they want to have a child with (Mohapatra, 2013). Some women feel pressured to find a partner at the suitable age and have family. A survey of five hundred and sixty women younger than thirty four years old published in Cosmopolitan in 2014 shows that over half of the interviewed women state that egg freezing takes off the pressure to find a partner in a short amount of time, allows them to have fun before starting a family, and to pursue a successful career (Pinto, 2017). Without the pressure to find a partner in their prime reproductive age, women have more time to find the most suitable partner (Mohapatra, 2013). Other benefits of egg freezing include allowing women to focus on self-improvement and decrease the risk of birth defects by using younger eggs (Mohapatra, 2013). The number of women who have their first child in their thirties and forties has quadrupled since 1970, but the number of women who have their child in their twenties declined by one third. With more women choosing to postpone their motherhood, egg freezing will become more popular. To date, two thousand babies have been born using frozen eggs worldwide and the numbers have been increasing (Mohapatra, 2013).

\subsection{Arguments Criticizing Egg Freezing}

Scholars also provided three convincing feminist approaches criticizing the technology of egg freezing. Cattapan first challenges the idea that egg freezing can allow women to "have it all" by eliminating the dilemma of pursuing a career and having biological children. While egg freezing does not ensure that women can "have it all": balancing between a perfect career and motherhood, it makes others think that achieving this balance is surely possible for women with the help of this technology. Egg freezing seems to be the "solution for all" but not solve the key issues women face. Making systematic efforts such as providing paid parental and sick leave, affordable childcare, comprehensive health insurance and adequate wages etc. can better empower women and encourage them to have children (Cattapan, 2014). Mohapatra states that if egg freezing becomes widely available in the workplace, women who decide to have a baby in their late twenties (normal time for having a baby) will be stigmatized as less desirable employees by the firms (Mohapatra, 2013). Moreover, after the delay of pregnancy, women still need reliable childcare and a paid maternity leave. Egg freezing is not a solution to these issues. Women pregnant at older ages might need more help and support than if she were young. It is also not clear whether an older woman will be able to return to the job after her delivery. According to Mohapatra, it is more critical for an employer to lose a senior employee due to maternity than a younger woman in the early stages of her career. Egg freezing is not promoting reproductive justice. Reproductive justice requires women to have social, political power to make choices that are healthy for their bodies in addition to the economic power. For employers, egg freezing is an easier solution compared to setting up childcare and maternity policies to relieve the pressure for women. But egg freezing is only a band aid that seems to cover the issue but not actually solve it (Mohapatra, 2013). In the debate of egg freezing, we should focus on the conception of women's autonomy. A true autonomous choice made by women should not be based on the constraints she faces in the workplace or with childcare. Providing women with relevant medical information does not mean that they have the full freedom of choice. If having a child at an earlier age means being stigmatized in the workplace and losing a career, a woman might not have her true autonomy when using egg freezing. The best way to solve this women's dilemma is to change the social norms rather than letting women adapt to these norms. It is not truly 
liberating that egg freezing takes off the pressure from women, the social constraints that are causing women to use egg freezing need to be examined. Why do women consider egg freezing as a good option? If workplaces are friendly to young women who plan to have kids, egg freezing would not be such an appealing option. There should be further research on women's motives to postpone motherhood and specific constraints they face every day in life (Shekdi-Rafid et al., 2012).

Egg freezing also gives women a sense of urgency that their fertile years are limited. Egg freezing focuses on extending a woman's fertile years by risky biomedical procedures which do not make childbearing easier for women (Cattapan, 2014). The concept of the "biological clock" originated in the 1970s to urge career women into becoming mothers by morally criticizing their hedonistic lifestyle (preferring their pleasures and sexual freedom to the responsibility of being mothers). The proliferation and commercialization of fertility treatments make the idea of "biological clocks" more prevalent and turn infertile women and their spouses into consumers. The "biological clock" is a mechanism employed by the reproductive industry to benefit itself. Seeing reproductive technologies like egg freezing as a control mechanism for women to live a better life connects to the concept of "medicalization" which means that the human experience and life cycles can be redefined as medical problems. This view gives the medical industry power (Shekdi-Rafid et al., 2012).

The technology and marketing of egg freezing reinforces the idea that women must have children. Egg freezing tries to ensure that women have the option to become mothers. And the ability to ensure motherhood might generate a moral imperative for women to engage in egg freezing to fulfill their responsibility of bearing children. The society will view women's inability to control the future by not freezing their eggs as their failure and responsibility alone (Cattapan, 2014).

\section{Social Review: Egg Freezing in the Context of Chinese Culture, Gender and Reproduction}

The Chinese government asserts control over women's reproductive rights with its policies, as it states in the slogan "a harmonious family is the cornerstone of a harmonious society" (Larson, 2012, 94-98). It had done so before with the implementation of one-child policy which forced many women to have abortions and be sterilized. With the Assisted Reproductive Technology Act that bans egg freezing for all single Chinese women and healthy married women in China, the government encourages women to marry and to have children at what it sees as the appropriate age. China needs more marriages and a higher birth rate to alleviate the pressure of an aging population, which is a result of the one-child policy. The government wants more young people to pay taxes and contribute to the economy so it can pay for the aging population's pension. To boost the birth rate, the Chinese government changed the one-child policy to two-child policy in 2016, allowing all families to have two children (AFP staff, 2019). With the intention of boosting the birth rate, the government cannot risk having universal access to egg freezing in China, because egg freezing would allow more young women postpone their motherhood and cause the already decreasing birth rate to drop again.

According to Article thirteen of the Chinese Assisted Reproductive Technology Act passed in 2001, it is prohibited to implement human assisted reproductive technology (including egg freezing) for couples who do not comply with the national population and family planning regulations and single women (Baidu). In other words, single women and healthy couples who do not suffer from illness or cancer that might cause infertility do not have access to egg freezing in China. Even couples who suffer from infertility need to provide the hospital with a marriage certificate and proof that they are not violating the one-child policy (or current two-child policy) to freeze eggs. In the context of how reproduction and single women are viewed in the Chinese culture, it is not surprising that the Chinese government implemented this policy to prevent most Chinese women from accessing egg freezing.

\subsection{The Trend of Marriage and Reproduction in China}

The Chinese culture has always valued marriage and reproduction. Families consider having offspring as continuing the family line and paying filial piety to the ancestors. Anxiety about marriage pervades through every aspect of contemporary Chinese culture. Many families with sons buy apartments or cars to make their sons more attractive in the marriage market. One of China's most popular TV shows is the dating show Fei Cheng Wu Rao (Don't Bother If You Aren't The One), in which a bachelor faces inquisition from twenty four young women standing behind lighted podiums. Chinese Elle slide show "Love guide: Eight Types of men who Shengnv love the most in 2012"and Chinese Bazar's article "From senior Shengnv to Queen of the wedding veil" both show that marriage is constantly encouraged and discussed by the media (Larson,2012,94-98). With a booming economy, women in urban China are marrying later, and the most educated women marry the latest, or sometimes not at all. In 1982, just five percent of urban women at the ages of twenty-five to twenty-nine were unmarried. By 1995, the percentage doubled. By 2008, the percentage nearly tripled. The number of women who are unmarried in their thirties, though still a small number, is increasing as well. Only two percent of women between thirty to forty years old were unmarried in 1995. By 2008, it was six percent. These statistics show that China's educated women increasingly know how they want to live their lives and who to get married with. But these rising standards make finding Mr. Right harder (Larson, 2012). The mean age at first marriage in China also continues to increase, from about seventeen point five years old around 1930 to eighteen point five years old in the 1940s, around 
twenty years old in 1970 and around twenty three years old in 1980. The increase of the age of first marriage has reduced the fertility rates in China. The total number of births from 1950 to 1981 was seven hundred ninety million, but if the age at marriage remained constant, there would have been an additional one hundred and four million births. The fertility rate from 1970 to 1980 was two hundred eighty-two million and without the increasing marriage age, it would have been sixty million more (Coale, 1989).

Women with high education degrees in China are increasingly rejecting the institution of marriage. In 2005, seven percent of forty-five-year-old Shanghai women with college degrees had never married. This phenomenon has resulted in a common joke that there are three genders in China: men, women, and women with Ph.D. 's who don't marry. This trend is also seen in other East Asian countries beside China. Twenty seven percent of college educated women aged 40 to 44 were single in Singapore. As a developing country with a fast-growing economy and expanding cities, it is reasonable to suspect that China will evolve in the same direction (Larson, 2012).

The delayed marriages and decrease in reproduction made many traditional Chinese parents very anxious. This caused the proliferation of "marriage markets" in many large Chinese cities including Shanghai, Chengdu and Guangzhou. "Marriage markets" are a unique sight in China. On Sundays in Beijing's Zhongshan Park, thousands of parents and grandparents gather to find matches for their children at the "marriage market". These elderly family members advertise for their child's eligibility as spouse by making signs for them usually containing their child's physical attributes, material possession and requirements for a partner. The signs are displayed along the sidewalks. Interested families meet and speak with each other to arrange meetings or dates for their children (Vandenberg, 2018). The ads at marriage markets usually portray men and women differently. Women were described as tender, charming or open-minded and men as responsible with a good paying job. These descriptions show that the Chinese culture views a good marriage as a wife caring for the family and the husband earning money (Jason, 2014). Parents usually enter the marriage market for similar reasons, either their children are too busy with their career to find a date or they haven't been successful with their relationships. They also suffer from the same kind of anxiety. Parents of daughters feel pressured because they don't want their daughters to be called the derogatory term of "leftovers" while parents of sons are worried because of China's gender imbalance (more men than women) (Vandenberg, 2018). Marriage markets can be seen as an offline dating app for anxious Chinese parents. It offers them a platform to make matches and seek opportunities for their unmarried children. Together the parents soothe each other's anxieties and try to find the perfect match (Vandenberg, 2018).

\subsection{How Single Women Are Viewed and Treated in China}

The phrase "sheng nv(剩女)", or "leftover ladies" refers to women above the age of twenty-seven, who are unmarried and considered "leftover" by the society, too old to be desirable wives. The topic of leftover women is increasingly discussed by the Chinese newspapers, TV programs and even government organizations like the All-China Women's Federation (Larson, 2012). The All-China Women's Federation (ACWF) is the official, state-sponsored organization representing women's interests in the People's Republic of China. The mission of the All-China Women's Federation, which was founded on April 3, 1949, is to represent the rights and interests of Chinese women and promote gender equality in China (Cheng, 2016). Ironically, the Chinese government utilized the organization to disseminate the derogatory term of "leftover ladies". The All-China Federation of Women used to have more than fifteen articles on its website on the subject of leftover women, ranging from giving advice on how to stand out, matchmaking tips and physiological analysis of why women want to marry late. An excerpt from an article called, Leftover Women Do Not Deserve Our Sympathy, posted on the website of the All-China Federation of Women in March 2011 wrote, "Pretty girls do not need a lot of education to marry into a rich and powerful family... Girls hope to further their education in order to increase their competitiveness. The tragedy is, they don't realize that as women age, they are worth less and less. So by the time they get their MA or PhD, they are already old - like yellowed pearls"(Magistad, 2013). To pressure unmarried women, the organization further assigned single women to labels like "leftover fighters" (twenty five to twenty seven years old), "the ones who must triumph"(twenty eight to thirty years old), and "master class of leftover women"(thirty five years old or older). These labels convey the message that women should get worried and get married at a young age (Larson, 2012, 94-98).

Wide-spread portrayals of "leftover ladies" by the government and other media sources caused the Chinese society to stigmatize unmarried women especially those above the age of twenty-seven. According to a survey by the All-China Women's Federation in 2010, more than ninety percent of male respondents agreed with the statement that women should get married before twenty-seven, or risk being undesirable (Larson, 2012). Ironically, there is a sharp contrast in perception and description of the scorned "leftover women" in China and the "individualized, liberated" women in the Western World. Western women's late marriage is usually referenced to their "individualization", a term that signifies the empowerment of women as they now have freedom to make more informed relationship choices, including whether to get married or not. Unlike in China, highly educated women who are not married are not stigmatized by society, but rather perceived as independent and liberated (To, 2013). 
With the rapid expansion of college education since 1999 in China, women have surpassed men in enrollment and graduation (Qian et al., 2014). The parents of the highly educated women grew up during the cultural revolution (1966-1976). During the era of chaos, they suffered a lack of educational opportunities. Thus it is understandable that they try their best to give their children the best education that they can afford. They believed that strong academic credentials could ensure the competitiveness of their children in the capitalist post-reform society. With the one child policy, families treat daughters and sons more equally and are willing to give their daughters good educational opportunities (To, 2013).

Some experts in China believe that women's rising standards are to be blamed for the delayed marriages and them becoming "leftovers". Wu Di, a contributor of China's Cosmopolitan and author of the book I Know Why You're Leftover, states that the increasing number of "leftover ladies" in China is caused by women's rising standards. As Wu said, "Today, women have more wealth, education which allows them to have better jobs and thus higher requirements for men. Now Chinese women want to find men they have deep feelings for and also own a house and a car. But not a lot of women can find men like that." Wu further encouraged women to want less and bear the shortcomings of their husbands. If women cannot do so, they will be single and "leftovers" (Larson,2012, 94-98).The Chinese Ministry of Education (2007) further attributes the unmarried status of highly educated women to their "overly high expectations for marriage partners", implying that they are the ones to blame for their independence and competence. This shows the patriarchal Chinese culture that still advocates the male as superior marriage style which causes independent and educated women to be discriminated against in the marriage market. The Chinese culture lacks positive descriptions of independent women who do not fit into the traditional roles in marriage. Marriage is seen as a "must" for Chinese women which results in marriage parks in local parks across China and nationwide matchmaking reality shows (To, 2013).

Other aspects of the Chinese culture including "the male superior norms", persisting gender roles in marriage, and parent's control also contributed to the difficulty for educated women to find a suitable partner. Many highly educated women had been rejected by their male romantic partners due to their strong economic accomplishments (To, 2013, 1-20). Men in their thirties are more likely than their female counterparts to marry someone less educated and younger (Qian et al., 2014). The "male superior norms" in the Chinese culture is the cause of educated women being "leftovers" in the marriage market. Some educated women try to avoid this problem by looking for "open minded" western men who are more relaxed about upholding the traditional "male superior norms". Another way for women is to conceal her accomplishments such as education level from potential suitors so they would not reject them due to the fear that they would be overshadowed by their potential wives (To, 2013)

Moreover, the traditional family roles still persisted in today's Chinese culture (To, 2013). The breadwinner role of the husband and the homemaker role of the wife remain in place. Urban women's domestic responsibilities are reinforced by the expectation for them to raise the "perfect child" under the one-child policy. Career oriented, independent women are "selfish, unfeminine and irresponsible", while husbands' failure to be the breadwinner of the family is seen as the main source of family conflict. Thus, the marriage pool for educated women is very small due to the shortage of highly educated men who do not feed into the "breadwinner mindset" (Qian et al., 2014.) In contrast, women who were subjected to the gendered role were offered marriage proposals or long-term relationships by their partners on the condition that they either quit their jobs after marriage or play the domestic role in the relationship. When such unequal requirements were raised by their suitors, some women choose to give up their marriage chances despite their urge to get married. Not all women face such extreme patriarchal demands such as quitting their jobs, but some also encountered control over their social life, work schedules and spending patterns. Veronica, a twenty-nine-year-old marketing manager said "I am very busy, and after work I need to go to lots of media events or parties, sometimes I need to go and meet friends. He (her boyfriend) would call me and ask why I am always out. I said I am not the type of girl who would stay at home and wait for you." This kind of segregated gender roles in marriage pose problems for educated, independent women (To, 2013, $1-20)$.

Chinese parents today still have considerable control over their daughter's marital lives due to the Chinese pre-modern era tradition of parents arranging women's marriages. The aspects of marriage that parents most want to interfere with were the economic status of the women's partner, the ethnicity of their partner, and the women's decision to get married. The majority of parents are traditional, they want their daughters to get married instead of pursuing relationships such as cohabitation or being single. Their expectation is to find partners who are of Chinese descent and who had higher economic status to be the main breadwinner of the family, which sometimes clashes with women's independent choices. For instance, educated women who chose Western partners hoping that they could be more accepting of their accomplishments might incite disagreement from their parents who preferred Chinese men. Many parents also have high expectations for the economic status of their daughter's partners. Chinese parents traditionally value "mendang hudui" (similar backgrounds) or raise their daughter's standard of living by "marrying up". Today, parents on one hand financed their daughter's education, making them more economically competitive, but on the other hand they still expected their daughters to fit into the traditional roles by leaving the main breadwinning role to their husbands. This showed parents' 
conflict when they embrace traditional values towards women's marriage and modern values toward advocating women's higher education (To, 2013).

The immense difficulty in finding a partner and stigmatization in society caused many single Chinese women to experience great pressure. Many single women over the age of twenty-five dread the idea of going home for the holidays because they are scolded by the elderlies for being single and have to endure relentless matchmaking efforts. Around eighty five percent of twenty-six to thirty-year-old unmarried Chinese women say that their parents or relatives urge them to get married according to a survey by Zhenai.com, a dating site in China. In an interview with The Washington Post, Emily Liu, a thirty-one-year-old single woman who works in a state-owned enterprise, talks about her anxiety of returning to her hometown Dalian and facing her parents." "I was so afraid last year that I didn't go home. I don't want to go back this year either, but there is no way to avoid it. My parents always tell me 'Your classmates already have children, but you don't even have a boyfriend.' Marriage is the only topic when I go back home, and my parents even mobilize my relatives to pressure me" (Fifield et al., 2019).

However, merely escaping from home and the nagging parents does not solve the problem. The core reason behind Chinese parent's growing anxieties is China's broader culture that values reproduction and sees marriage as women's responsibility (Fifield et al., 2019).

\subsection{A Generational Divide on Perception of Egg Freezing in China}

Based on the discussions of the pressure and social stigmatization single women face in China, it is not surprising that both older and younger generations of Chinese women alike hold contradictory views on the technology of egg freezing. The older generations influenced by the Chinese cultural belief of marriage and reproduction as women's responsibility reject the practice of egg freezing. For many parents seeking opportunities for their children in the "marriage markets" at local parks or constantly scolding their single children for not getting married, marriage means settling down and passing down the family line. These rooted beliefs have also contributed to the stigmatization of single women over twenty-seven in the Chinese society and the wide-spread usage of the derogatory term "leftovers". With their rooted belief of seeing marriage and reproduction as women's responsibility, the older generation's anxiety increases with the access of egg freezing as a new option for single women to delay childbirth and marriage.

Contrary to their parents, young women in China embrace the practice of egg freezing. With the age of first marriage continuing to increase in China and more young women receiving higher education, many consider the option of egg freezing to focus on their careers and find a suitable partner (Vandenberg, 2018). College education and education abroad allow many young women to embrace the ideal that women should not rely on men. They began to rebel against the "male superior norms" and parent involvement in marriage which is rooted in Chinese culture. To them, a single life offers more opportunities to build their careers and enjoy themselves without the constraints of motherhood. Many young women also have their own standards of finding a suitable partner. Even in their thirties, many women do not want to hastily get married with someone of the same economic background or even marry up to a richer man as the older generations have done. Egg freezing is a weapon to help them fight against their traditional parents and social stigma surrounding single women in China. When the stigmatized group of single women in China make autonomous decisions to utilize egg freezing, it helps open reproductive freedom.

Overall, Chinese people have different perceptions of egg freezing and it is important to understand and analyze their opinions. Answering a well-designed survey allows people to candidly express their opinions on egg freezing. By analyzing these opinions, I can know the extent of understanding Chinese have on egg freezing and the specific reasons why they support or reject this technology. The analysis further gives readers a big picture of how Chinese perceive egg freezing and the current Chinese egg freezing policy. I am also able to provide information on aspects of egg freezing that Chinese people lack knowledge on.

\section{Survey on Chinese Citizens' Opinions on Egg Freezing}

\subsection{Methodology}

I created a survey to assess Chinese people's opinions on egg freezing within a broadly changing reproductive climate. I designed the survey on the Tencent survey app. The survey includes a short introduction to the technology of egg freezing to provide people with relevant background information. The survey consists of eleven questions, including two demographic questions on the gender and age of the respondent, two multiple choice questions regarding how much they feel they know about the technology of egg freezing, and seven open-ended questions where respondents could express their own opinions. I distributed the survey using Wechat, the most widely used social media in China. In addition to posting the survey on my own account, I posted it on my feminist public account "Discover" that has about two thousand followers. I opened the survey for five days and gained fifty-two responses. I analyzed the collected data by organizing them in excel to find a common theme. 
Survey Questions:

1. What is your gender?

2. What is your age?

3. Have you ever heard of the technology of egg freezing?

4. Do you know anyone (your relatives/ friends/ parents etc.) who have used egg freezing?

5. How well do you think you know egg freezing? (rate from 1 to 10 )

6.What are your feelings about egg freezing?

7.What do you think are the advantages of egg freezing?

8. What do you think are the disadvantages of egg freezing?

9. Do you think single Chinese women should have access to egg freezing? Why?

10. Do you think married Chinese women who does not suffer from infertility should have access to egg freezing? Why?

11. What questions do you have about egg freezing?

地域位置

Shanghai

Guangdong

Jiangsu

Beijing

Shandong

Others
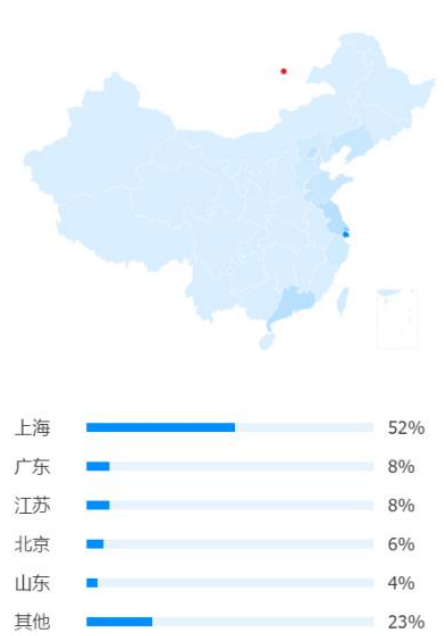

\section{Demographic distribution}

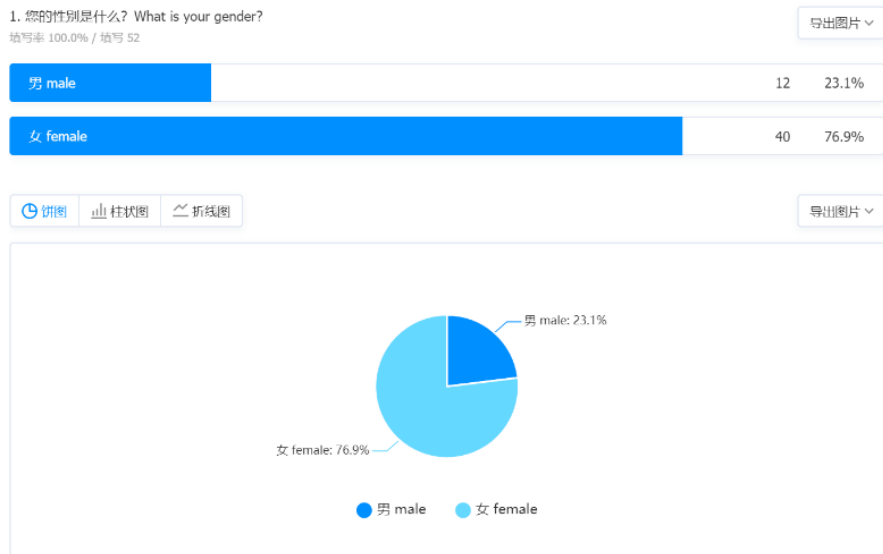

Question 1 distribution 


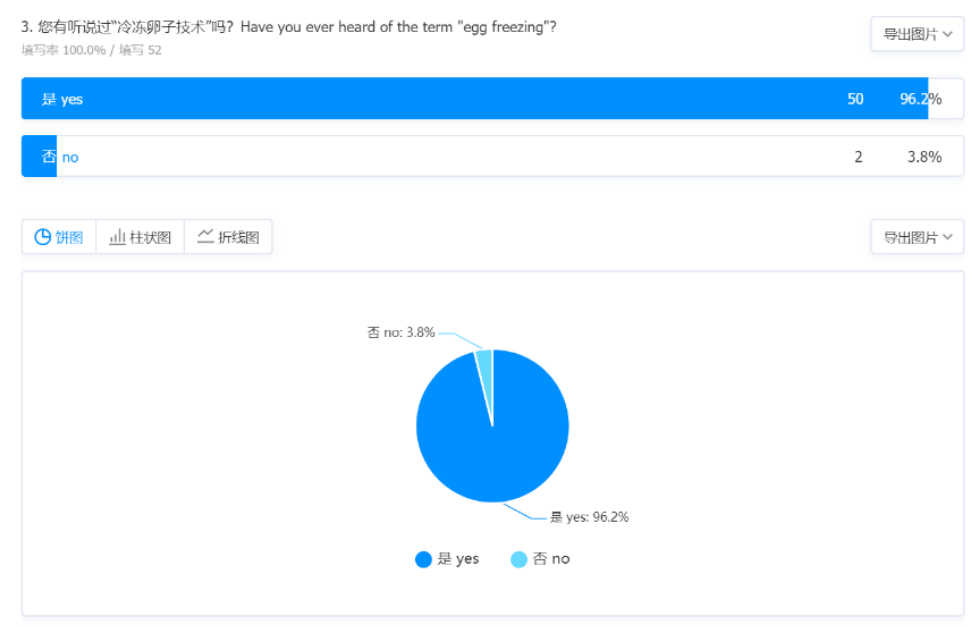

Question 3 distribution

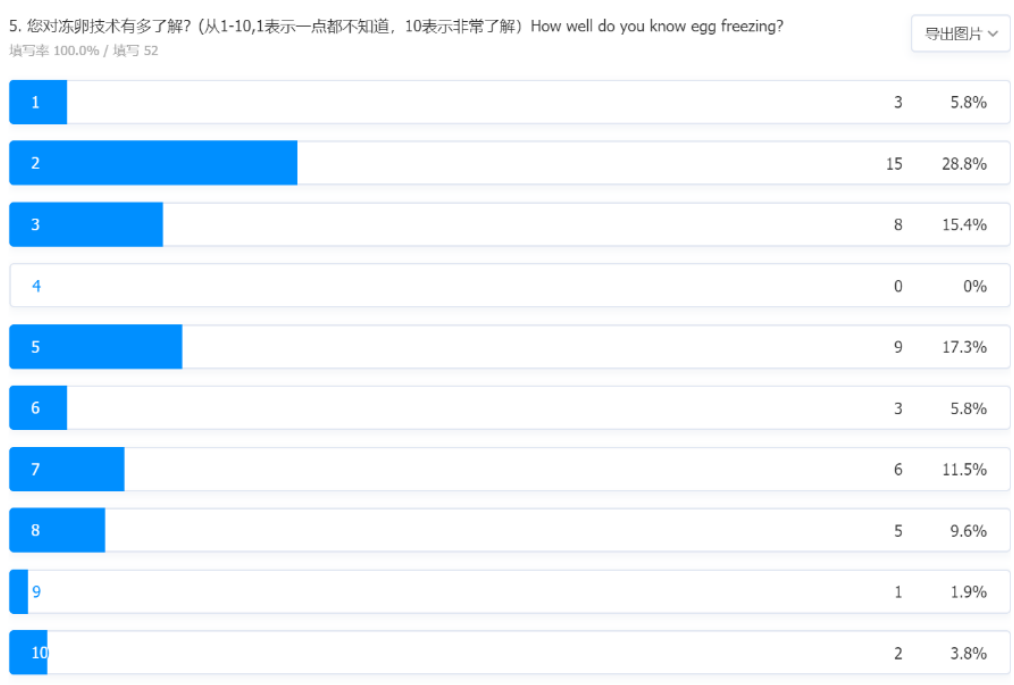

Question 5 distribution

\subsection{Results from Survey on Chinese Citizens' Opinions on Egg Freezing}

Respondents included Chinese people aged fifteen to fifty-five. Seventy-seven percent of respondents are female, and twenty three percent are male. This gender disparity in respondents might suggest that overall Chinese women pay more attention and are more interested in the technology of egg freezing. This lack of interest on behalf of Chinese men might be caused by the Chinese culture which sees reproduction as a women's responsibility, and men as less involved. Fifty two percent of respondents are in Shanghai, and other fourteen percent are in big cities such as Beijing and Guangdong. This might suggest that Chinese people in big cities are more exposed to advanced technologies and feminist media (public accounts).

Ninety-six percent of respondents have heard of the term egg freezing. Despite respondents' varying degree of understanding of egg freezing, only one out of fifty-one respondents answered "no" for the question: "Do you think Chinese single women should have access to egg freezing?" Similarly, only four respondents answered "no" for: "Do you think married Chinese women who don't suffer from infertility should have access to egg freezing?" This shows that the survey respondents generally hold very liberal views on egg freezing. It is interesting to note that even though most respondents hold liberal views, most questions raised by respondents in the section "What questions do you have about egg freezing?" are centered on the health risks of egg freezing and how it impacts the fetus. These questions reveal that most respondents lack basic information on the risks, success rate and cost of egg freezing. Without knowing this information, it is hard to make a fair judgement, and most respondent's reason to have universal access to egg freezing is based on the vague understanding of "women have liberty to control their body and make choices". 


\section{Discussion}

To address the questions raised by respondents in the survey, I provide scholarly research on the health risks, cost and moral issues of egg freezing from a global perspective in this section. The dangers of egg freezing are usually ignored by the public and avoided in the commercials of the fertility clinics (Cattapan, 2014). Knowing the risks and disadvantages of egg freezing help people make informed decisions. Egg freezing requires women to undergo hormone treatments to stimulate egg production and undergo surgery to retrieve those eggs which are frozen for later use. The procedure of egg retrieval has many risks including headaches, bloating and abdominal pain. In extreme cases of ovarian hyperstimulation, there can be severe pain, vomiting, fluid buildup and in rare cases death. There is limited oversight of the in/fertility industry in the US and Canada, which is not necessarily true for China. And no longitudinal research has been done on the health effects of the procedure. In addition, women face the risks of undergoing pregnancy at an advanced reproductive age which includes increased risks of miscarriage, ectopic pregnancy, preeclampsia, gestational diabetes, placental complications, intrauterine growth restriction, and caesarian section. Without stating these risks, the promotion of egg freezing downplays the personal, social, and physical costs of this technology (Cattapan, 2014). It is important to note that when the American Society for reproductive medicine (ASRM) took off the experimental label on egg freezing in 2013, it also stated "There are not yet sufficient data to recommend oocyte cryopreservation [egg freezing] for the sole purpose of circumventing reproductive aging in healthy women because there are no data to support the safety, efficacy, ethics, emotional risks, and cost-effectiveness of oocyte cryopreservation for this indication" (Pinto, 2017, 107-126).

Using egg freezing also does not ensure that there will be a successful pregnancy. The media framed egg freezing as a revolutionary technology similar to the contraception pill that gives them "an insurance" to postpone childbirth. Some clinics claim to have forty to sixty percent success rate. However other experts are concerned that the success rate is misleading and has caused women to place too much hope in egg freezing. Most research suggests that the success rates are between five to ten percent, much lower than what people believe (Aire, 2015). A survey of fertility clinics in the US offering egg freezing shows that nearly half of them do not have experience in using thawed eggs to result in pregnancies. With little experience, the thawed eggs might fail to result in a pregnancy when the woman wants to. In addition, the current studies are done on eggs that have been frozen for a short period of time. So it is not clear how long-term freezing would affect the success rate of pregnancies from thawed eggs (Mohapatra, 2013).

Considering the uncertainties and risks, egg freezing is shockingly expensive. This expensive technology has the potential to exacerbate the class difference between rich and poor women. Egg freezing is not a realistic option for working class women and delayed fertility is increasingly being associated with the rich and educated women (Mohapatra, 2013). With the estimated cost of between seven thousand dollars to ten thousand dollars per cycle in the United States and approximately five thousand CAD to ten thousand CAD in Canada, egg freezing is not an accessible solution for women who do not have a lot of money which disproportionately includes many women of color, single women, and sexual minority women. The procedure is much cheaper in China than in North America: from early check, ovulation induction, egg retrieval to embryo transplantation, the treatment for one patient cost thirty thousand to fifty thousand yuan (four thousand two hundred to seven thousand sixty US dollars) (Chen, 2020). Only egg freezing is even cheaper, but it remains inaccessible to most women in China with the current policy. There is also the annual storage cost of between two hundred euros and four hundred euros and the cost of thawing the eggs and subsequent in vitro fertilization (IVF) for the pregnancy. Unlike celebrities who can afford this procedure, few women have thousands of dollars available to freeze their eggs. Reproductive technologies like egg freezing are developed for white, heterosexual, cisgender, married couples with substantial savings. Sexual minority women are ignored in this picture, for they are not experiencing infertility as it is traditionally and biomedically defined. They are socially infertile. Due to discrimination and lack of funds, many sexual minority women have trouble accessing reproductive technologies. It is imperative to include voices of sexual minority women, single women, and low-income women in the debate of egg freezing (Cattapan, 2014).

Egg freezing also raises moral issues. Women's eggs are important for stem cell research and other scientific research areas. Therefore the need for women's eggs in scientific research might open up a transnational market for surplus eggs from egg freezing. This could raise moral and social questions for women freezing their eggs: how to measure the value of the eggs and should eggs be exchanged for money? These kinds of underground human egg transactions can cause women's bodies not only to be seen as biological clocks but also biological mines which can be used for research (Cattapan, 2014). Rashly providing universal access to egg freezing without oversight brings serious health and moral issues.

Besides the lack of understanding on the disadvantages of egg freezing, a large number of survey respondents wrote in answers such as: "egg freezing allows women to give birth when they are ready" and "egg freezing extends fertile years to allow women to postpone pregnancy" to the question of "What are the advantages of egg freezing?". Some even wrote "egg freezing helps boost birth rate", "infertile women can have children and be happy". These answers have a similar theme of seeing "preservation of motherhood" as the main purpose and advantage of egg freezing. This idea reinforces the 
Chinese traditional view that reproduction is women's responsibility, thus generating a moral imperative for women to engage in egg freezing to fulfill their responsibility of reproducing (Cattapan, 2014). Instead of fighting against the patriarchal culture, egg freezing is supporting one of its major viewpoints. As discussed above, single women in China are already stigmatized as "undesirable, unfeminine" by the government, and many people bought into it due to the culture that values reproduction. When egg freezing becomes widely available, Chinese women can be forced to use this technology to ensure that they will become mothers, just like how single women over the age of twenty-seven are pressured to get married today.

\section{Conclusion}

The Chinese government has a long history of controlling Chinese women's reproductive rights. When the government implemented the one-child policy in the late 1970s to reduce the population, forced abortions and sterilizations on women were widely performed across China. The government heavily fined those who disobeyed the policy, and most lost their jobs (Pletcher, 2020). This paper critically examines the current government policy that bans egg freezing for single women and healthy married women and situates the policy within a broader discussion of reproductive freedom and control in China. By analysing the global debate on egg freezing and the different perceptions of the technology in China, I conclude that egg freezing is a complex technology that seems to open up reproductive freedom but can also be used to restrict women's reproductive rights. To truly liberate women, egg freezing must be women's autonomous decision, which means that they are fully informed of the advantages and risks of the technology and are not pressured by their family or the society to use it. I encourage women to gain thorough knowledge about the pros and cons of egg freezing and the current government policy in the country. When Chinese women have the knowledge to make their autonomous decision, they can choose to protest the government's policy or make amendments to it. I hope that this paper can inspire more research on reproductive rights and development of feminism in China with the goal of educating Chinese women.

\section{Acknowledgements}

The author would like to thank my friends who are also interested in feminist issues for supporting me throughout the process of writing this paper. My friends give me motivation when I was making edits and revising this paper. It could not be done without the mental support.

\section{Conflict of Interest}

I do not have a conflict of interest when writing this paper. This paper is solely based on my own interests and observations, no personal or financial interests influenced my position in this paper.

\section{References}

AFP staff AFP. (2019). Single Chinese Woman Sues over Egg Freezing. CTV News.

Aire, S. A. (2015). Is Too Much Hope Placed in Egg Freezing? BMJ: British Medical Journal, 351, JSTOR. https://doi.org/10.1136/bmj.h5955

Anonymous. (2019). Chinese Woman Sues Hospital for Refusing to Freeze Her Eggs. BBC News, BBC.

Anonymous. Egg Freezing. UCLA, www.uclahealth.org.

Baidu. Assisted Reproductive Technology Act.

Cattapan A. C. (2014). Breaking the Ice: Young Feminist Scholars of Reproductive Politics Reflect on Egg Freezing. International Journal of Feminist Approaches to Bioethics, 7(2), 236-247, Jstor.

https://doi.org/10.3138/ijfab.7.2.0236

Chen X. C. (2020). Should China's Single Women Be Allowed to Freeze Eggs? CGTN.

Cheng, E. C. (2016). All-China Women's Federation. Encyclopædia Britannica, Encyclopædia Britannica.

Coale, A. C. (1989). Marriage and Childbearing in China since 1940. Social Forces, 67(4), 833-850, Oxford University Press, Jstor. https://doi.org/10.2307/2579704

Fifield, A. F., \& Liu, L. Y. (2019). For 'Leftover' Single Women in China, Lunar New Year Brings Dread of Facing Their Families. The Washington Post, WP Company.

Hilgers, L. H. (2015). Chinese Actress Comes to America to Freeze Her Eggs, Internet Goes Wild. Foreign Policy.

Jason. (2014). Shenzhen Marriage Park: Want Ads of Last Resort. The Ugly Wife Is a Treasure at Home: True Stories of Love and Marriage in Communist China, by Melissa Margaret Scheinder, University of Nebraska Press, 2014, pp. 131-142, Jstor. https://doi.org/10.2307/j.ctt1d9nn9w.23

Kuo, L. K. (2019). Single Woman Sues Chinese Hospital for Refusal to Freeze Eggs. The Guardian, Guardian News and Media. 
Larson, C. L. (2012). The Startling Plight of China's Leftover Ladies. Foreign Policy, 193, 94-98, Jstor.

Magistad, M. M. (2013). China's 'Leftover Women', Unmarried at 27. BBC News, BBC.

Mitch, M. L. (2007). Melting Opposition to Frozen Eggs. Science, 316(5823), 388-389, Jstor. ttps://doi.org/10.1126/science.316.5823.388

Mohapatra, S. M. (2013). Using Egg Freezing to Extend the Biological Clock: Fertility Insurance or False Hope? SSRN.

Pinto-Correia C. P. (2017). Technological Motherhood: Fear, Wonder, and Science in the New Age of Reproductive Biotechnology. Columbia University Press, Jstor. https://doi.org/10.7312/columbia/9780231170949.003.0006

Pletcher, K. P. (2020). One-Child Policy. Encyclopædia BritannicaInc.

Qian, Y. Q., \& Zhenchao, Z. Q. (2014). The Gender Divide in Urban China: Singlehood and Assortative Mating by Age and Education. Demographic Research, 31, 1337-1364, Jstor. https://doi.org/10.4054/DemRes.2014.31.45

Schulte, B. S. (2014). Freezing eggs, or freezing moms out of high-tech?Washington Post.

Shkedi-Rafid, S. S., \& Yael, H. Y. (2012). Egg Freezing for Non-Medical Uses: the Lack of a Relational Approach to Autonomy in the New Israeli Policy and in Academic Discussion. Journal of Medical Ethics, 38(3), 154-157, Jstor. https://doi.org/10.1136/medethics-2011-100088

To, S. T. (2013). Understanding Sheng $N u$ ('Leftover Women'): The Phenomenon of Late Marriage among Chinese Professional Women. Symbolic Interaction, 36(1), 1-20, Jstor. https://doi.org/10.1002/symb.46

Vandenberg, L. V. (2018). Finding Love in China's Marriage Markets. The Diplomat.

\section{Copyrights}

Copyright for this article is retained by the author(s), with first publication rights granted to the journal.

This is an open-access article distributed under the terms and conditions of the Creative Commons Attribution license which permits unrestricted use, distribution, and reproduction in any medium, provided the original work is properly cited. 